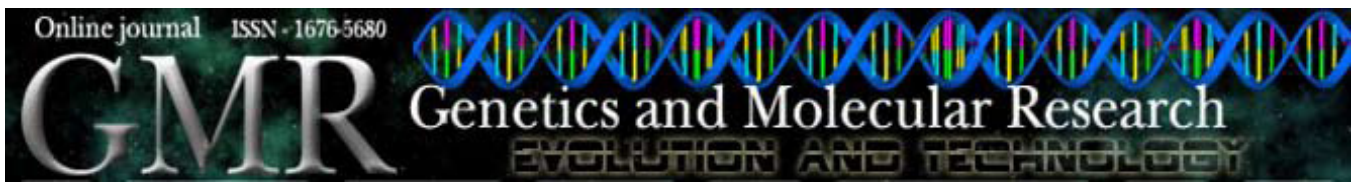

Thesis Abstract

\title{
Spermatogenesis and nucleolar behavior in males of aquatic Heteroptera
}

\author{
M.M.U. Castanhole
}

2009. Instituto de Biociências, Letras e Ciências Exatas, Universidade Paulista Júlio de Mesquita Filho, São José do Rio Preto, SP, Brasil. Master's thesis. Orienting professor: Dr. Mary Massumi Itoyama

Aspects of spermatogenesis and nucleolar behavior were analyzed in Brachymetra albinerva, Cylindrostethus palmaris, Halobatopsis platensis, Limnogonus aduncus (Gerridae), Martarega sp (Notonectidae), Rhagovelia whitei, and Rhagovelia sp (Veliidae). The testicles are rounded (Veliidae), elongated (Gerridae) or spiral (Notonectidae) and have a transparent membrane covering them. The complement chromosome was $2 \mathrm{n}=23(22 \mathrm{~A}+\mathrm{X} 0$, L. aduncus and Rhagovelia sp), $25(24 \mathrm{~A}+\mathrm{X} 0$, B. albinerva and H. platensis $), 26(22 \mathrm{~A}+2 \mathrm{~m}+\mathrm{XY}$, Martarega sp), 29 (28A + X0, C. palmaris), or 39 (38A + X0, R. whitei) chromosomes, and the only species with a different sex chromosome system was Martarega sp, which showed an $\mathrm{XY}$ system and m-chromosomes. The meiotic behavior of all species was similar: holocentric chromosomes and heteropyknotic material at prophase, interstitial and/or terminal chiasmata, and first reductional division for the autosomes and the reverse for the sex chromosomes. The only difference observed was related to the very large size of Martarega sp cells in all stages of spermatogenesis. With regard to nucleolar behavior, the species did not show differences, except for Martarega sp with larger nucleoli than the other species. The only species in which it was clearly possible to identify the nucleolar organizer region was L. aduncus, in the region of a terminal autosome. It was also confirmed that the telomeric associations do not occur at random. In the other species, specific staining was very discrete, and the nucleolar organizer region location was not at all evident.

Key words: Aquatic Heteroptera; Gerridae; Notonectidae; Veliidae; Meiosis; Nucleolar behavior 